\title{
Integration of multilayered graphene on AlN based resonators as a functionalization platform for biosensors
}

\author{
L. Gordillo-Dagallier, J. Olivares, B. Marco-Dufort, J.L. Olivera, J.M. Escolano, T. Mirea, M. Clement, and E. Iborra
}

\begin{abstract}
In this communication we present the integration of graphene on the top electrode of solidly mounted resonators based on piezoelectric AIN thin films for gravimetric sensor applications. Because its particular chemical structure, graphene shows great potential as a bio-functionalization platform. How ever, in order to be commercially viable, direct deposition on the resonators is preferred to transfer techniques. The high temperatures involved in graphene chemical vapor deposition make this integration complex. Specially developed acoustic resonators, which can withstand high temperatures, combined with the use of multilayered top electrodes for AIN resonators, allow a successful integration of graphene. Finally, oxygen plasma treatments to optimize the creation of defects on the graphene layer can be used to firmly bond receptors on its surface.
\end{abstract}

Keywords-Graphene integration; $B A W$ resonators; gravimetric sensors; surface functionalization

\section{INTRODUCTION}

Applications for graphene are studied in a variety of fields; for sensors, graphene is demonstrating very good possibilities. For instance, in the field of chemical sensors, the particular chemical structure of graphene makes it especially interesting for the surface functionalization of biosensors [1]. Graphene layers on top of devices can be obtained by several means, which include the direct transfer of already synthesized graphene layers as well as chemical vapor deposition (CVD) techniques. Transfer methods allow obtaining a graphene layer on virtually any surface. However, the existing transfer methods are usually complex and not reproducible. Additionally, the binding of transferred graphene to the surface is not always tight enough to withstand a subsequent functionalization process, for which several incubations and rinsing stages are usually needed. CVD graphene has the advantage of better adhesion. However, it needs a particular (catalytic) surface to grow. Transition metals such as Ni, Co, Ir, Mo or W have been the object of study. The graphene growth process depends on the nature of the catalyst, concretely on the competition between $\mathrm{C}$ - $\mathrm{C}$ bonding and $\mathrm{C}$ metal bonding [2]. For the particular applications, biofunctionalization, graphene should have some particular features that are not the commonly pursued ones. First, the adherence of graphene to the top electrode should be as tight as possible because the basis of gravimetric detection needs the added mass to coherently vibrate with the resonator. Besides, acoustic energy could detach the layer. Secondly, during the functionalization process, several reagents are used to covalently bind the receptor species to the surface. Therefore, some chemical reactions that debilitate the graphene structure might occur. To ensure that this does not detach the graphene film, it is better to have two or even three graphene layers instead of the always-pursued monolayer structure. The control of the deposition process, including the choice of the particular catalyst metal, is very important in this case, as it determines the characteristics of the whole structure. Another issue to take into consideration is that, usually, temperatures as high as $1000^{\circ} \mathrm{C}$ are needed for high quality mono-layered graphene deposition, although lower temperatures (down to $600^{\circ} \mathrm{C}$ ) can be used if the appropriate catalyst and process conditions are chosen, but, even in these last cases, temperatures are still very high. The devices on which the graphene layer is deposited must withstand these temperatures without losing their performances. For instance, metals can be oxidized, semiconductors can be doped, and compounds can be decomposed. Additionally, the changes in sizes due to dilatation can generate stress between layers of different materials, which can create defects or even delamination if the adherence is not very good. In the particular case of solidly mounted resonators (SMRs), which are composed of a piezoelectric AlN capacitor deposited on a layered acoustic reflector containing five or more piled layers, the main issue is to preserve the mechanical integrity of the whole structure, and he its functionality. Due to the different thermal expansion coefficients of the materials involved in the multi-layered structure, in-plane stress develops during CVDgraphene deposition, which may lead to the delamination of the multi-layered structure. It is important to choose adequately the materials and their deposition conditions to minimize the thermal expansion and improve the adhesion between layers.

In this paper, we present the integration of graphene on AlNbased SMRs as a functionalization platform for chemical sensors. We describe a procedure to manufacture SMRs containing acoustic reflectors specifically designed to be withstand the high temperatures (up to $1000^{\circ} \mathrm{C}$ ) to which the samples are subjected during the CVD growth of graphene. We describe the process for obtaining low-defect and few-layer graphene on top of the SMR. 


\section{EXPERIMENTAL}

SMRs are composed of Ir/AIN/Mo piezoelectric stacks deposited on acoustic Bragg reflectors made of nine alternate $\mathrm{SiO}_{2}$ and AlN layers a quarter-wavelength thick (see Fig. 1). All the films were deposited by sputtering. The deposition conditions of the high impedance (AIN) and low impedance $\left(\mathrm{SiO}_{2}\right)$ layers of the reflector were adjusted to minimize the residual stress of each film. After deposition, the reflectors were subjected to heat-treatments under vacuum at different temperatures to assess their temperature resistance. Then, the piezoelectric stack was deposited with both $c$-axis oriented and tilted-c-axis AlN films. A proper design combined with lowstress materials yielded SMRs capable of sustaining temperatures as high as $1000^{\circ} \mathrm{C}$ without losing their performance.

SMRs with a $\mathrm{W}$ top electrode were also used because this material has a high acoustic impedance and is commonly used in resonators. Moreover, a Ti thin film under and over the top electrode was tested, as Ti is a typical adhesion between metals.

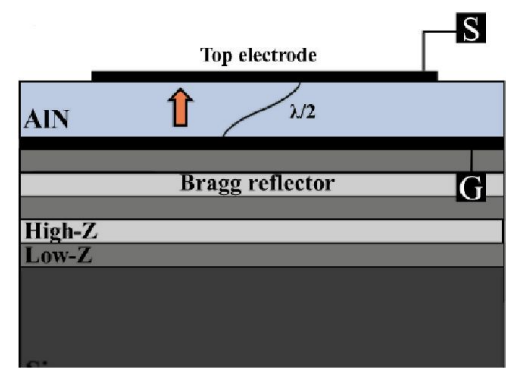

Fig. 1. AlN-based SMR

A thin film of catalyst polycrystalline nickel (50-120 nm) was thermally evaporated over the Mo top electrode for subsequent graphene deposition. The use of polycrystalline $\mathrm{Ni}$ is attractive due to its low cost compared to single crystalline $\mathrm{Ni}$, and therefore more suitable for industrial commercialization. Ni films had to be thin enough not to interfere with the resonator performance, but thick enough to avoid the formation of $\mathrm{Ni}$ droplets on the surface during heating due to dewetting [3], which could deteriorate graphene quality or promote CNT growth if nanoparticles are formed. Figure 2 shows an AFM image showing the surface of a representative sample after graphene deposition on a $100 \mathrm{~nm}$-thick Ni layer.

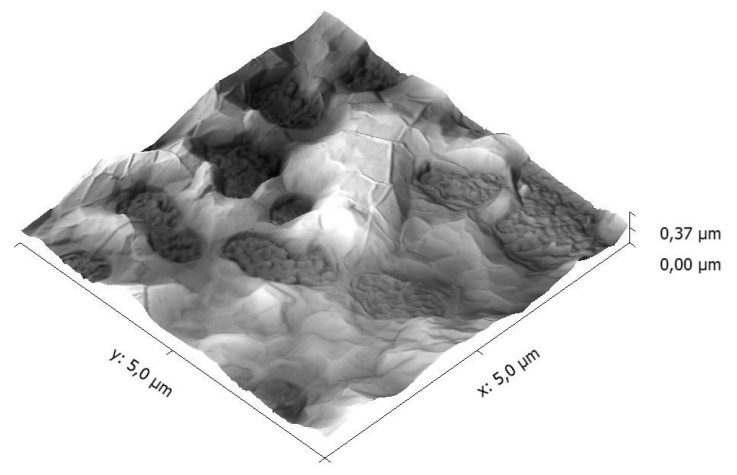

Fig. 2. 3D-AFM image of the droplets formed in $\mathrm{Ni}$ surface due to $\mathrm{Ni}$ dewetting from the substrate. Observe the fold structure of the graphene on the Ni surface.
Graphene was grown in a high vacuum custom-made CVD chamber on top of the Ni catalyst deposited on the SMRs. The chamber was pumped down to a pressure of $10^{-6}$ Torr with a turbo-molecular pump before starting the depositions. A pyrolytic carbon heater was used as a substrate holder to decompose diluted $\mathrm{C}_{2} \mathrm{H}_{2}$ gas (acetylene) at a low temperature $\left(650^{\circ} \mathrm{C}\right)$. The total pressure in the chamber was kept relatively high and constant ( $\sim 10 \mathrm{mTorr})$. The SMRs were first heated in argon at a rate of $50^{\circ} \mathrm{C} / \mathrm{min}$ up to $650^{\circ} \mathrm{C}$, in order to minimize the height of the Ni droplets formed. They were then annealed for $4 \mathrm{~min}$ in a mixture of $\mathrm{Ar}$ and $\mathrm{C}_{2} \mathrm{H}_{2}$ ( $18 \%$ by volume). Finally, the SMRs were slowly cooled down at a rate of $15^{\circ} \mathrm{C} / \mathrm{min}$ in pure Ar from 650 to $600{ }^{\circ} \mathrm{C}$ and then rapidly cooled to room temperature (Fig. 3). The graphene obtained on Ni-Mo and $\mathrm{Ni}-\mathrm{W}$ top electrodes was compared with the graphene grown on $\mathrm{Ni}-\mathrm{SiO}_{2}$ samples, in order to determine the influence of the under-layer in the characteristics of the obtained graphene.

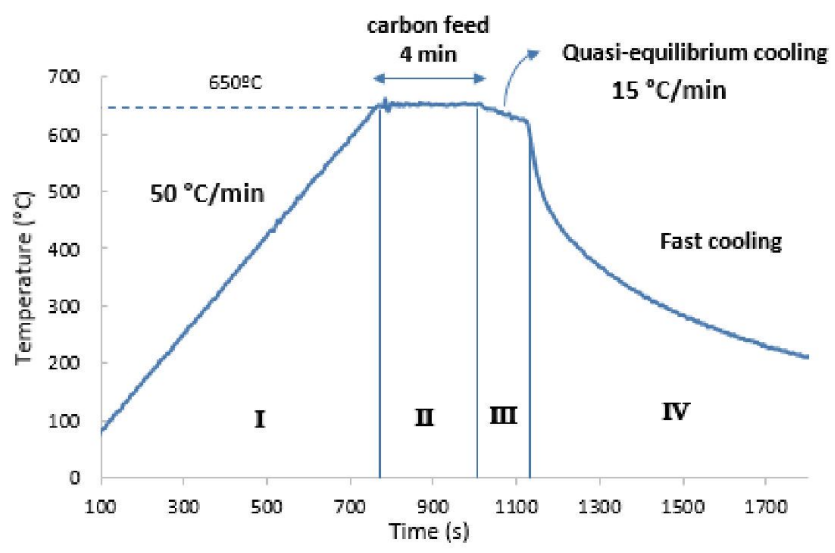

Fig. 3. Temperature evolution during the graphene growth process. Four stages are represented, corresponding to the sample heating (I), the $\mathrm{C}_{2} \mathrm{H}_{2}$ supply (II), the slow cooling (III), and the fast cooling (IV) stages.

The CVD-grown graphene obtained on top of the SMRs was characterized using Raman spectroscopy with an excitation light of $532 \mathrm{~nm}$ wavelength. Multiple measurements were taken throughout the samples to assess the uniformity and quality of the coverage. Then, the SMRs performance was determined by deriving the electrical impedance spectrum from the reflection coefficient $\left(S_{11}\right)$ measured with a network analyzer.

Finally, as a first step for surface functionalization, a low damage oxygen radical plasma treatment was used to generate controlled defect in graphene. The optimal parameters of operation were $30 \mathrm{~W}$ of RF power, a pressure of $25 \mathrm{~m}$ Torr and an $\mathrm{O}_{2}$ flow of $25 \mathrm{sccm}$. In order to protect the sample from the energetic ions associated with plasmas, a glass shield was placed on top of the samples, only allowing thermalized reactive oxygen radicals to reach the surface and thus protecting the graphene from heavy damage [4]. The samples were characterized using Raman spectroscopy before and after each plasma treatments (of different durations) for monitoring the amount of defects and the integrity of the graphene layer.

\section{RESULTS AND DISCUSSION}

The main characteristics of the CVD-grown graphene are the low level of defects and the presence of two or three layers 
weakly coupled. A typical Raman spectrum is shown in Fig.4 in blue, with the characteristic D peak $\left(\sim 1350 \mathrm{~cm}^{-1}\right)$, which is related with defects in the graphene structure, G peak $(\sim 1580$ $\left.\mathrm{cm}^{-1}\right)$ and 2D peak $\left(\sim 2700 \mathrm{~cm}^{-1}\right)$. The number of graphene layers can be inferred from the $I_{2 D} / I_{G}$ ratio, which is $I_{2 D} / I_{G}>1$, and the analysis of the $2 \mathrm{D}$ peak, which is well fitted with a single Lorentzian peak and has a FWHM of around $40 \mathrm{~cm}^{-1}$ [5]. Moreover, this fitting suggests that the interlayer coupling is weak [6].

Higher $\mathrm{C}_{2} \mathrm{H}_{2}$ flow rates and lower overall pressures, as well as quick variations of total pressure, result in an increased $\mathrm{D}$ peak (see Fig. 4a). Thus, given a $\mathrm{C}_{2} \mathrm{H}_{2}-\mathrm{Ar}$ flow ratio, raising the total pressure and keeping it constant during the growth process show to be an effective method to control the number of defects in the as-grown graphene. However, when the overall pressure is raised over a threshold, no graphene grows. Furthermore, slowing the cooling rate until reaching $600^{\circ} \mathrm{C}$ delivers graphene with better quality, greater coverage and uniformity, and fewer layers, as can be deduced from the increased $\mathrm{I}_{2 \mathrm{D}} / \mathrm{I}_{G}$ ratio in Fig. 4b. Nevertheless, if the cooling rate is slowed until lower temperatures $\left(<500^{\circ} \mathrm{C}\right)$, no graphene growth is observed.
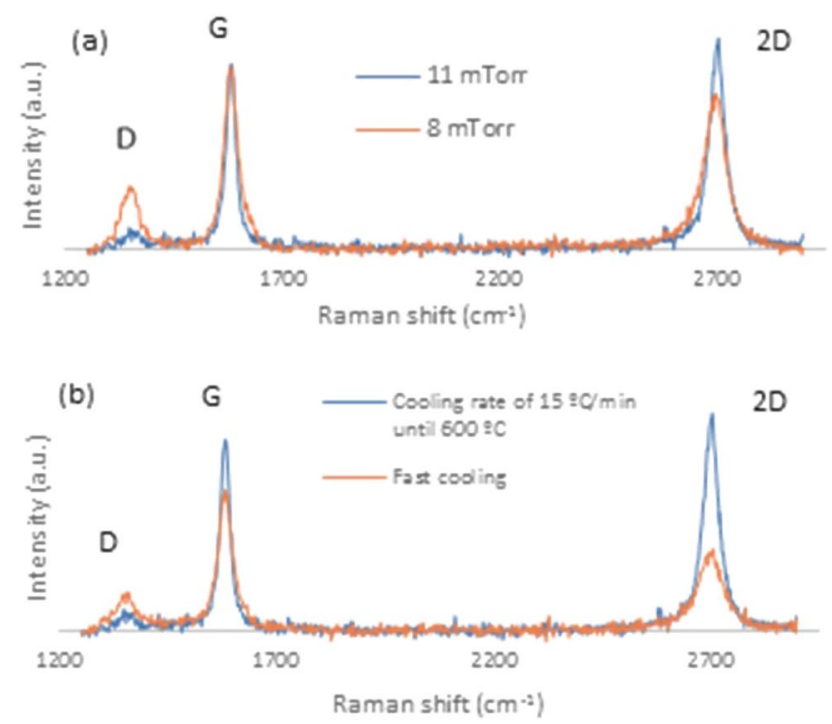

Fig. 4. (a) Comparison of the Raman spectra of graphene grown upon fast cooling and with a cooling rate of $15^{\circ} \mathrm{C} / \mathrm{min}$ from 650 to $600^{\circ} \mathrm{C}$. (b) Influence of overall pressure during growth process.

The nature of the under-layer of the Ni catalyst was found to have a major influence on the quality of the as-grown graphene. The results shown above correspond to Ni deposited directly on the Mo top electrode. With a $\mathrm{W}$ top the graphene quality obtained is similar. However, when introducing even a very thin Ti film between the Ni and the Mo, graphite and nickel carbides were grown. An explanation to this results is proposed below. The hydrocarbon gas precursor $\left(\mathrm{C}_{2} \mathrm{H}_{2}\right)$ is decomposed at $650^{\circ} \mathrm{C}$ catalytically on the Ni surface (see stage II in Fig. 3) and carbon diffuses into the Ni bulk. It is believed that graphene then grows following a combination of two mechanisms: carbon segregation from the $\mathrm{Ni}$ bulk under thermal equilibrium conditions at carbon concentrations below the solubility limit, and direct surface non-equilibrium carbon precipitation during quenching [7] (see Fig. 3). The surface curvature associated with the presence of grain boundaries and defects on the polycrystalline Ni substrate act as active sites for multilayer graphene nucleation [8]. Once a particular nucleation site is activated, it fulfils the carbon atoms for the growing graphene from the surrounding grains. The graphene layers then grow laterally from the nucleation site and cover the Ni surface [7]. Due to these phenomena, there is an equilibrium between carbon diffusion into the Ni bulk and carbon segregation through the active nucleation points (graphene construction and destruction). By acting upon this equilibrium (stages II and III in Fig. 3) and controlling carbon precipitation during fast cooling (stage IV in Fig. 3), it is possible to obtain high quality few-layered graphene even on polycrystalline $\mathrm{Ni}$. While the equilibrium can be controlled by regulating the $\mathrm{C}_{2} \mathrm{H}_{2}$ flow rate, the duration of the $\mathrm{C}_{2} \mathrm{H}_{2}$ supply, and the overall pressure and temperature process; the carbon precipitation upon rapid cooling can be limited if an adequate substrate is chosen.

On one hand, by keeping the pressure constant during the whole growth process, disturbances in the equilibrium are minimized and low number of defects are induced during the graphene crystallization. Higher overall pressures may slow carbon segregation, resulting in a more controlled process, and thus obtaining graphene with lower defects and higher quality. On the other hand, by decreasing the cooling rate, this equilibrium segregated phase is kept until $600{ }^{\circ} \mathrm{C}$. As reported by Reina et al. [9], decreasing the cooling rate reduces the number of multi-layer graphene nucleation sites, and therefore increases the Ni surface covered by few-layers. Moreover, by diluting the $\mathrm{C}_{2} \mathrm{H}_{2}$ in $\mathrm{Ar}$, the amount of carbon in solution into the Ni bulk is controlled, and therefore the amount of carbon precipitation upon cooling. Once $600^{\circ} \mathrm{C}$ was reached, fast cooling allows to freeze the graphene structure resulting in a full few-layered graphene coverage. Regarding the influence of the under-layer, a Mo layer under the Ni acts as a carbon sink, retaining dissolved excess carbon species in the form of molybdenum carbides, as suggested in [10]. However, a Ti layer acts as a diffusion barrier. As the carbon is retained near the surface by the diffusion barrier, it can segregate easier back to it, resulting in multi-layer graphene.

The theory exposed above has been proven to be valid for different catalysts as graphene has been grown successfully on $\mathrm{Fe}$ and $\mathrm{Co}$. In order to extrapolate the growth process parameters to obtain similar graphene quality, the solubility of carbon in the catalyst must be taken into account. If the solubility in the new catalyst is lower than in $\mathrm{Ni}$, growth times and $\mathrm{C}_{2} \mathrm{H}_{2}$ flow rates must be increased.

To check whether the performance of the SMRs was affected by the integration of the graphene, the electrical impedance spectra of the SMRs before and after graphene deposition was measured, as shown in Fig. 5. The slight variation in frequency, also observed after a heat treatment without graphene deposition, is due to variations of thicknesses of the different layers, which became more compact. The quality factor and the electromechanical coupling coefficient do not substantially vary. Therefore, it was concluded that the integration of the graphene does not significantly modify the performance of the SMRs. 


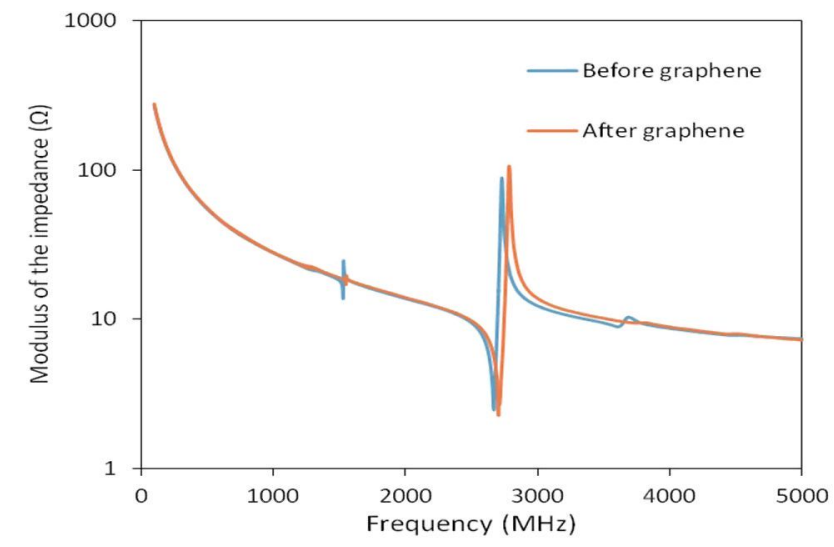

Fig. 5. Modulus of the impedance spectra of a device before and after the deposition of graphene on the top electrode.

After successful integration of the graphene on the SMRs, its surface was functionalized for bio-detecting demonstration. To do this, shear mode resonators made with tilted AlN grains were used for in liquid operation [11]. Functionalization starts with surface exposition to an $\mathrm{O}_{2}$ plasma used to create oxygencontaining defects on the graphene surface. This is evidenced in Fig. 6, which shows Raman spectra before and after a plasma treatment. After the plasma treatment, the D peak - or defect peak - appears at around $1350 \mathrm{~cm}^{-1}$. The intensities of the $\mathrm{G}$ and 2D peaks are unaffected by the plasma treatment, thus, we can conclude that the plasma treatment successfully introduced defects in the graphene while maintaining its structural integrity.

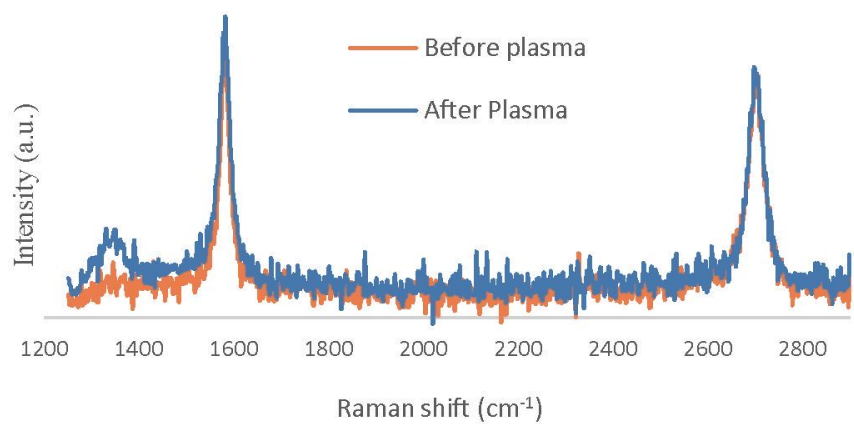

Fig. 6. Raman spectra of the few-layered graphene surface of an SMR before and after the low damage $\mathrm{O}_{2}$ treatmen. Note the appearance of the defect D peak at around $1350 \mathrm{~cm}^{-1}$ after the plasma treatment.

After the plasma treatment, streptavidin was bonded to the surface of the graphene on top of the SMRs. A protocol was developed to covalently bond Streptavidin to oxygen-containing defects on the surface of the graphene, using standard EDC/NHS (1-ethyl-3-(3-dimethylaminopropyl) carbodiimide hydrochloride/N-Hydroxysuccinimide) chemistry. To characterize this union, streptavidin conjugated to Qdot $\AA$ 655v was used and fluorescence microscopy was employed to see the fluorescence signal. The red signal shown in Fig. 7(a) evidences the uniform distribution of streptavidin-Qdot on top of a graphene sample (positive control). Figure 7(b) shows a negative control in which EDC/NHS was omitted.

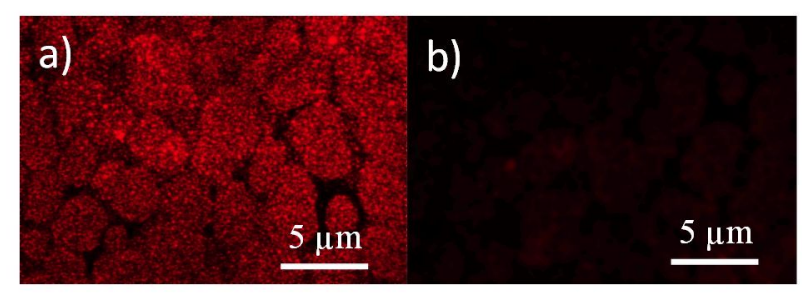

Fig. 7. Fluorescence microscopy images of the surface of graphene incubated with streptavidin conjugated to Qdot $\mathbb{B} 655$, a) positive and b) negative controls. The underlying texture of the graphene is visualized.

\section{CONCLUSIONS}

Graphene directly grown by CVD was integrated on SMR as a functionalization platform for chemical sensing. After developing devices able to withstand the high process temperature, few-layered graphene was grown on them. A model of the graphene growth, which can be extended to other catalysts, is proposed. The performance of resonators is not degraded by graphene growth. $\mathrm{O}_{2}$ plasma treatment allows control of defect formation on the graphene layer. The viability of SMR with graphene was demonstrated.

\section{ACKNOWLEDGMENT}

This work was partially supported by MICINN del Gobierno de España through project MAT2013-45957-R.

\section{REFERENCES}

[1] D.J. Joe, et al., "Surface Functionalized Graphene Biosensor on Sapphire for Cancer Cell Detection", J Nanosci Nanotechnol. 16(1) (2016) pp. 144151.

[2] H. Chen et al., "Contrasting Behavior of Carbon Nucleation in the Initial Stages of Graphene Epitaxial Growth on Stepped Metal Surfaces", Phys. Rev. Lett. 104 (2010), 186101.

[3] R.G. Golik, et al., "Analysis of Structural Transformations and Fluctuation Effects in the Nanosized Nickel Films in the Vicinity of the Melting Point", Journal of Communications Technology and Electronics 57(6) (2012) pp. 629-633.

[4] C-H Huang, et al., "Ultra low-damage radical treatment for the highly controllable oxidation of large-scale graphene sheets", Carbon 73 (2014) pp.244-251.

[5] A. C. Ferrari, "Raman Spectroscopy of graphene and graphite", Solid State Communications 143 (2007) pp. 47-57.

[6] Z. Luo, et al., "Large-Scale Synthesis of Bi-Layer Graphene in Strongly Coupled Stacking Order", Adv. Funct. Mater. 21 (2011) pp. 911-917.

[7] L. Baraton, et al., "On the mechanisms of precipitation of graphene on nickel thin films", Europhysics Letters 96(4) (2011).

[8] Y. Zhang et al., "Comparison of Graphene Growth on Single-Crystalline and Polycrystalline Ni by Chemical Vapor Deposition", J. Phys. Chem. Lett. 1 (2010) pp. 3101-3107.

[9] A. Reina et al., "Growth of Large-Area Single- and Bi-Layer Graphene by Controlled Carbon Precipitation on Polycrystalline Ni Surfaces", Nano Res 2 (2009) pp. 509-516.

[10] B. Dai, et al., "Rational design of a binary metal alloy for chemical vapour deposition growth of uniform single-layer graphene", Nature Communications 2 (2011).

[11] Mario DeMiguel-Ramos, Bárbara Díaz-Durán, José-Miguel Escolano, Mariano Barba, Teona Mirea, Jimena Olivares, Marta Clement, Enrique Iborra, "Gravimetric biosensor based on a $1.3 \mathrm{GHz}$ AlN shear-mode solidly mounted resonator". Sensors and Actuators B 239 (2017) 1282 1288. 